(C) The Authors 2015. This is an Open Access article, distributed under the terms of the Creative Commons

Attribution-NonCommercial-NoDerivatives licence (http://creativecommons.org/licenses/by-nc-nd/4.0/),

which permits non-commercial re-use, distribution, and reproduction in any medium, provided the original

work is unaltered and is properly cited. The written permission of Cambridge University Press must be obtained

for commercial re-use or in order to create a derivative work.

\title{
Longitudinal association of dairy consumption with the changes in blood pressure and the risk of incident hypertension: the Framingham Heart Study
}

\author{
Huifen Wang ${ }^{1}$, Caroline S. Fox ${ }^{2}$, Lisa M. Troy ${ }^{3}$, Nicola M. Mckeown ${ }^{1}$ and Paul F. Jacques ${ }^{1 *}$ \\ ${ }^{1}$ Jean Mayer US Department of Agriculture (USDA) Human Nutrition Research Center on Aging, Tufts University, \\ 711 Washington St, Boston, MA O2111, USA \\ ${ }^{2}$ National Heart, Lung, and Blood Institute's Framingham Heart Study, Framingham, MA 01702, USA \\ ${ }^{3}$ Department of Nutrition, Chenoweth Laboratory, University of Massachusetts, Amberst, MA 01003, USA
}

(Submitted 16 June 2015 - Final revision received 27 July 2015 - Accepted 12 August 2015 - First published online 23 September 2015)

\section{Abstract}

We aimed to examine the longitudinal association of dairy consumption with the changes in blood pressure (BP) and the risk of incident hypertension (HTN) among adults. This study included 2636 Framingham Heart Study Offspring Cohort members who participated in the 5th through 8th examinations (1991-2008) and were free of HTN at their first examination during the follow-up. Data collected at each examination included dietary intake (by a validated FFQ), BP (following standardised procedures) and anti-hypertensive medication use (by physician-elicited self-report). HTN was defined as systolic BP (SBP) $\geq 140 \mathrm{mmHg}$, or diastolic BP (DBP) $\geq 90 \mathrm{mmHg}$ or anti-hypertensive medication use. We used repeated-measure and discrete-time hazard regressions to examine the associations of dairy consumption with the annualised BP change ( $n$ 2075) and incident HTN ( $n$ 2340; cases =1026), respectively. Covariates included demographic, lifestyle, overall diet quality, metabolic factors and medication use. Greater intakes of total dairy foods, total low-fat/fat-free dairy foods, low-fat/skimmed milk and yoghurt were associated with smaller annualised increments in SBP and a lower risk of projected HTN incidence. However, with the exception of total dairy foods and yoghurt, these inverse associations with HTN risk were attenuated as the follow-up time increased. For yoghurt, each additional serving was associated with 6 (95\% CI 1, 10) \% reduced risk of incident HTN. Total dairy and total low-fat/fat-free dairy intakes were found to be inversely related to changes in DBP. Dairy consumption, as part of a nutritious and energy-balanced diet pattern, may benefit BP control and prevent or delay the onset of HTN.

\section{Key words: Dairy intake: Blood pressure: Hypertension: Milk products: Yoghurt}

Owing to the well-established risk associated with hypertension $(\mathrm{HTN})^{(1)}$, the prevalence of HTN presents significant threats to public health in the $\mathrm{USA}^{(2)}$ and throughout the world ${ }^{(3)}$. The lifetime risk of developing HTN has been estimated to be almost $90 \%{ }^{(4)}$. The importance of lowering blood pressure (BP) for reducing stroke and myocardial infarction risk is also clearly demonstrated $^{(5,6)}$. A $5 \mathrm{mmHg}$ reduction in BP has been found to be associated with a $21 \%$ lower risk of IHD and $34 \%$ lower risk of stroke ${ }^{(7)}$.

Dietary modification can be an effective strategy for HTN prevention $^{(8)}$, and dairy products may be a critical component of that strategy ${ }^{(9)}$. The Dietary Approaches to Stop Hypertension Trial found that low-fat/fat-free dairy foods added significant benefits to vegetables and fruits in lowering $\mathrm{BP}^{(9)}$. Randomised clinical trials conducted to specifically examine whether dairy intake influences BP are inconclusive ${ }^{(10,11)}$. This is likely a result of their relative short study duration (mostly up to several weeks) and small sample size.
On the other hand, recent reviews and meta-analyses of prospective cohort studies (with follow-up durations ranging from 2 to15 years) ${ }^{(10,12,13)}$ have suggested that milk (irrespective of fat content) and low-fat dairy products may facilitate the prevention of HTN. Although the meta-analyses also concluded that the findings for total high-fat dairy products or fermented dairy products are variable and overall no significant association was observed in relation to HTN incidence ${ }^{(13)}$, evidence is limited in quantity and quality. Additional evidence is warranted. Moreover, the possibility of different health effects of dairy fats as opposed to that in other animal foods (e.g. meat) remains largely unstudied and open to debate; very few studies have reported adverse health effects or associations with highfat dairy consumption ${ }^{(13-16)}$. Greater dairy consumption may also correlate with healthier dietary patterns ${ }^{(17-19)}$, but not all existing studies of dairy consumption and BP or HTN have accounted for potential confounding by overall diet quality $^{(20,21)}$. Finally, most of the existing longitudinal studies

Abbreviations: BP, blood pressure; DBP, diastolic BP; DGAI, Dietary Guidelines Adherence Index; FHS, Framingham Heart Study; HTN, hypertension; SBP, systolic BP.

*Corresponding author: P. F. Jacques, fax +1 6175563344 , email Paul.jacques@tufts.edu 
that examined the association between dairy intake and incident HTN only assessed dietary intake once at baseline. However, failing to account for the potential changes in dairy intake over long follow-up periods as well as changes in other health characteristics (such as weight) may weaken the relationship between dairy intake and HTN incidence. Therefore, the present study aimed to assess the consumption of various high-fat and low-fat dairy products in relation to the change in BP and the development of incident HTN accounting for overall diet quality. We used repeated measures of health, lifestyle and dietary factors (including dietary intake and overall diet quality) to assess changes in diet, health and lifestyle over an average 15-year follow-up.

\section{Methods \\ Study population}

We used data from participants involved in the 5th (1991-1995), the 6th (1995-1998), the 7th (1998-2001) and the 8th (20052008) Framingham Heart Study (FHS) Offspring Cohort examinations $^{(22)}$. In brief, the original FHS followed a cohort of US adults (age range 28-62 years at baseline) since 1948 to study CVD and its risk factors. In 1971, 5124 offspring (aged 5-70 years) of the original FHS cohort were recruited to participate in the FHS Offspring Cohort Study. As of 2008, eight examinations have been conducted with response rates among survivors of $74.1 \%$ ( $n$ 3799), 68.9\% ( $n$ 3532), 69.1\% ( $n$ 3539) and $59.0 \%$ ( $n$ 3021) for examinations 5 to 8 , respectively. Of note, the examinations were not held at a regular interval, mainly due to the need to balance limited resources for data collection activities with the other Framingham cohorts (original and generation 3 cohorts).

At each examination, participants underwent a standardised medical history and physical examination, whereas dietary intakes were assessed from examination 5 to examination 8, inclusive. This study was conducted according to the guidelines laid down in the Declaration of Helsinki. All study protocols and procedures were approved by the Boston University Medical Campus Institutional Review Board. Written informed consent was obtained from all the participants. The present project was reviewed and approved by the Tufts Medical Center Institutional Review Board.

\section{Dietary assessments}

The structure and contents of the FFQ. A 126-item semiquantitative $\mathrm{FFQ}^{(23)}$ was mailed to the participants before each examination. Participants were asked to bring the completed FFQ with them during their examination visits. The FFQ queried participants on how often, on average, during the past year they consumed a standardised serving size of each food (e.g. $227 \mathrm{~g}$ of yoghurt, one $245 \mathrm{~g}$ glass of skimmed or low-fat milk, etc.). There were nine frequency categories on the FFQ, ranging from 'never or $<1$ serving/month' to 'more than 6 servings/d'. The FFQ Dairy section included the following: skimmed or low-fat milk, whole milk, cream (e.g. in coffee, whipped), sour cream, sherbet or ice milk, ice cream, yoghurt, cottage or ricotta cheese, cream cheese, other cheese (e.g. American, Cheddar, etc.), non-dairy coffee whitener, margarine and butter.

The relative validity of the FFQ has been reported previously $^{(23-25)}$. The FFQ was considered to be invalid when participants reported total energy intake of $<2510.4 \mathrm{~kJ} / \mathrm{d}$ (i.e. $<600 \mathrm{kcal} / \mathrm{d}$ ) for all or $>16736 \mathrm{~kJ} / \mathrm{d}$ (i.e. $>4000 \mathrm{kcal} / \mathrm{d}$ ) for women and $>17572.8 \mathrm{~kJ} / \mathrm{d}$ (i.e. $>4200 \mathrm{kcal} / \mathrm{d}$ ) for men or $>12$ blank food items. The validity of dairy intake from the FFQ has been assessed based on a comparison of the FFQ with two 7-d diet records collected during the year time interval covered by the $\mathrm{FFQ}^{(25,26)}$. For dairy products, the correlations between intakes assessed by the FFQ and by diet records were 0.81 for skimmed/low-fat milk, 0.62 for whole milk, 0.73 for ice cream, 0.80 for cottage cheese, 0.57 for hard cheese and 0.94 for yoghurt $^{(25,26)}$.

Processing FFQ data into database. For each food item, if participants reported consuming 'never or $<1$ serving/month', their intake of that food item was coded as zero servings/week in the FFQ database. If participants reported consuming '1-3 servings/month' or more of a particular food item, their intake of that food item was converted to 'servings/week' based on the mid-range value of the intake category. The daily nutrient values were calculated by multiplying the nutrient concentration of the specific portion size of each food by the daily consumption frequency and summing across all food items. The portion size of each dairy and milk product in the FFQ is as follows: 1 serving of skimmed/low-fat milk $=245 \mathrm{~g}$; 1 serving of whole milk $=244 \mathrm{~g} ; 1$ serving of cream $=15 \mathrm{~g} ; 1$ serving of sour cream $=12 \mathrm{~g} ; 1$ serving of sherbet/ice milk $=96 \mathrm{~g} ; 1$ serving of ice cream $=66 \mathrm{~g} ; 1$ serving of yoghurt $=227 \mathrm{~g} ; 1$ serving of cottage $/$ ricotta cheese $=105 \mathrm{~g} ; 1$ serving of cream cheese $=28 \mathrm{~g}$; 1 serving of other cheese (e.g. American, Cheddar, etc.) $=28 \mathrm{~g}$; and 1 serving of butter $=5 \mathrm{~g}$. All the foods were coded according to the US Department of Agriculture (USDA) Nutrient Database (NDB) ${ }^{(27)}$ based on the most representative products consumed in the USA at the time of examination.

Processing dietary intake data for the current analyses. For the present analyses, total dairy consumption was calculated by summing the servings per week of all the dairy foods, which include skimmed/low-fat milk (NDB no. 01085, 01079), whole milk (NDB 01077), sherbet/ice milk (NDB 19097), ice cream (NDB 19095), yoghurt (NDB 01121), cottage/ricotta cheese (NDB 01012) and other cheese (e.g. American, Cheddar, etc.) (NDB 01009). Similarly, the total low-fat dairy consumption was calculated by summing intakes of skimmed/low-fat milk, sherbet/ice milk and yoghurt. The total high-fat dairy products included whole milk, ice cream, cottage/ricotta cheese and other cheese (e.g. American, Cheddar, etc.). We examined skimmed/low-fat milk and whole milk separately because of their different fat concentrations, but we also created a fluid milk group by summing up the intakes of skimmed/low-fat milk and whole milk. Of note, according to the definitions by the USDA Food Groups ${ }^{(28)}$ and the Dietary Guidelines for Americans ${ }^{(29)}$, milk products with little to no Ca were not included in the Dairy Group. Therefore, we did not include 
cream (NDB 01050), sour cream (NDB 01056), cream cheese (NDB 01017) and butter (NDB 01001) in any of the dairy groups for the present analyses. However, for the purpose of comparison, we created a separate group 'Cream and butter' by summing the intake of these four milk products. In addition, we created a 'Fermented milk products' group, which included sour cream, yoghurt, cottage/ricotta cheese, cream cheese and other cheese (e.g. American, Cheddar, etc.).

The consumption of dairy and milk products was represented as servings per week, except for total dairy intake, which was expressed as servings per day. To estimate the usual dairy and milk products consumption within each exam interval, we averaged the usual intake reported at the beginning and at the end of the interval. This approach better captured the long-term dietary intake and minimised variation in dietary assessment. Based on their consumption of each dairy/milk food or group, participants were further categorised into three groups: $<1$ servings, 1 to $<3$ servings and $\geq 3$ servings/d for total dairy intake or per week for other dairy/milk foods or groups.

The Dietary Guidelines Adherence Index (DGAI), a score created to assess the adherence of participants to the key dietary recommendations by the 2005 Dietary Guidelines for Americans ${ }^{(30)}$, was used in the present study as a measurement of participants' overall dietary quality. As the consumption of milk and milk products and the likelihood of choosing low-fat milk and milk products constitute two of the DGAI's twenty items, we used a modified DGAI based on the remaining eighteen items to assess diet quality independent of dairy intake.

\section{Measurements of blood pressure and hypertension}

At each examination, sitting BP was measured twice by a physician on each participant after a 5 -min rest period using a random-zero sphygmomanometer, and two readings were averaged for the analyses. HTN medication use during the follow-up was ascertained via physician-elicited self-report. Incident HTN was identified at each examination defined as systolic BP $\quad(\mathrm{SBP}) \geq 140 \mathrm{mmHg}$ or diastolic $\mathrm{BP} \quad(\mathrm{DBP}) \geq 90$ mmHg or current use of anti-hypertensive medication ${ }^{(31)}$.

\section{Measurements of other variables}

Height (to the nearest $0.635 \mathrm{~cm}$ ) and weight (to the nearest $0.227 \mathrm{~kg}$ ) were measured with the participant standing, shoes off and wearing only a hospital gown. The scale was calibrated daily. BMI was calculated in $\mathrm{kg} / \mathrm{m}^{2}$. Standardised physical examination was conducted, and questionnaires were used to assess participants' lifestyle and medical history. A physical activity index (PAI) score, expressed in metabolic equivalents, was calculated by averaging the number of hours spent on specific activities (i.e. sleep, sedentary, slight activity, moderate activity and heavy activity) with each activity weighted by the $\mathrm{VO}_{2}$ required to perform the activity ${ }^{(32)}$. PAI was not available for examination 6 , and thus values from examination 5 were also used for examination 6 . Fasting $(\geq 8 \mathrm{~h}$ ) blood samples were drawn for assessing the levels of glucose and lipids. A hexokinase/glucose-6-phosphate dehydrogenase method ${ }^{(33)}$ was used to measure serum glucose. Plasma total cholesterol and TAG were measured by enzymatic methods ${ }^{(34)}$, and HDL-cholesterol was measured after dextran-Mg precipitation $^{(35)}$. For glucose, TAG, total cholesterol and HDLcholesterol, the intra- and inter-assay CV were all $<2$ and $<3 \%$, respectively ${ }^{(36)}$.

\section{Statistical analysis}

All the analyses were conducted separately for BP change and incident HTN across different dairy foods and groups using SAS statistical software (version 9.3; SAS Institute). All statistical tests were two-sided. Statistical significance was set at $P<0.05$. Skewed data were log-transformed before being entered for the analysis, as appropriate.

To maximise the sample size and minimise potential selection bias as a result of loss of surviving participants who did not attend all follow-up study examinations, the present longitudinal analyses overall included 2636 participants who attended at least one of the examinations 5 through 7 and were free of HTN at baseline - that is, excluding those who had prevalent HTN or were missing HTN data at baseline. We defined participants' baseline examination as their first appearance during examinations 5 through 7 . These participants contributed 10544 potential observations across exams.

\section{Association between the consumption of dairy/milk products and the annualised change in blood pressure. For} the analysis of BP change, we excluded 2358 out of 10544 observations due to missing or invalid FFQ data (including dairy consumption data) or missing BP data during follow-up. A total of 1066 observations included anti-hypertensive medication use and were excluded as well. After these exclusions, 459 participants had only one complete and valid observation. As the annualised change in BP could not be calculated for these 459 participants, they were also excluded from our final analyses, leaving 2075 participants with 6661 observations. Participants who were excluded from the analyses were slightly older and less healthy (e.g. higher cholesterol and TAG concentrations, etc.) than those who remained in the analyses (data not shown).

We used repeated-measure regression (PROC MIXED) to examine the longitudinal association between dairy consumption and annualised change in SBP and DBP within exam intervals across the follow-up. An unstructured variance structure was specified. The change in SBP and DBP was calculated separately for each participant as the difference between two non-missing and valid adjacent measurements. We used annualised change as the outcome variable for the current analyses to correct for the unequal time intervals between examinations.

As mentioned above, we averaged the usual dairy/milk consumption reported at the beginning and at the end of the interval to estimate the usual dairy consumption within each exam interval. Participants were then further categorised into three groups based on their consumption of dairy/milk products: $<1$ servings, 1 to $<3$ servings and $\geq 3$ servings/d for total dairy intake or per week for other dairy foods/groups. 
The average intake of total energy and food groups (i.e. fish, meat, whole grain, refined grain, alcohol, caffeine coffee and fruits and vegetables), as well as the average DGAI score within each exam interval, were also estimated in a similar way as described above.

Models were adjusted, as appropriate, for sex, and time-varying factors, including measurements of SBP or DBP at the beginning of each exam interval, age, physical activity, smoking status, BMI, diabetic status, the ratio of total cholesterol and HDL-cholesterol, TAG concentrations and the use of anti-hyperlipidaemia/ hormone-replacement therapy/oral contraceptive medications at the beginning of each exam interval and average total energy intake, caffeinated coffee intake and modified DGAI score within each exam interval. Linear trend of annualised BP change across dairy consumption groups was tested using the medium intake in each group.

In a sensitivity analysis of $\mathrm{BP}$ change, we repeated the above analyses by including 1066 observations with antihypertensive medication use during follow-up, which yielded 7921 observations for 2292 participants. This analysis was, thus, additionally adjusted for the anti-hypertensive medication use during follow-up. The percentages of participants who reported using anti-hypertensive medications were $8 \cdot 6,14.3$ and $32.4 \%$ at examinations 6-8, respectively.

\section{Association between dairy consumption and incident} hypertension. To examine the longitudinal association between dairy consumption and the development of incident HTN, 296 out of 2636 participants were excluded due to missing or invalid FFQ data (including dairy consumption data) at their baseline examination, leaving 2340 participants for the primary analysis

Discrete-time hazard regressions were used for the analysis with dairy consumption entered into models as continuous, time-varying variables. For participants who were censored or not found to develop incident HTN during the follow-up, we calculated survival time as the time from their baseline examination to the end of examination 8 (i.e. 31 December 2008). For participants who were found to have incident HTN during follow-up, we calculated survival time as the time from their baseline examination to the last examination without incident HTN plus half the time between the last examination without incident HTN and the examination at which incident HTN was identified. Usual dietary intake for each participant was updated during follow-up by averaging all non-missing and valid FFQ data before the onset of incident HTN or the end of the study. Other lifestyle and metabolic factors were also treated as timevarying covariates by using the latest non-missing records before the onset of incident HTN or the end of the study.

All the models were adjusted, as appropriate, for time-invariant sex and baseline age and time-varying covariates including physical activity, smoking status, BMI, diabetic status, the ratio of total cholesterol and HDL-cholesterol, TAG concentrations, the use of cholesterol-lowering medication, hormone-replacement therapy or oral contraceptives, caffeinated coffee intake, total energy intake and modified DGAI scores (excluding items related to consumption of milk and milk products and the likelihood of choosing low-fat milk and milk products). Of note, alcohol and $\mathrm{Na}$ intake are represented in the DGAI score, and thus were not additionally adjusted for in the regression models.

Proportional hazard assumption was tested, as appropriate, for all covariates and dairy consumption variables by including an interaction term between these variables and the follow-up time. A statistically significant interaction term indicates a violation of the proportionality assumption. In cases of proportionality violation, we included the interaction term in the model to correct for the non-proportionality.

We conducted a sensitivity analyses for examining the longitudinal association between dairy consumption and incident HTN by further excluding 130 out of 2340 participants who did not come back after their baseline examinations.

For both the analyses of annualised $\mathrm{BP}$ change and the development of incident HTN, we further examined the associations adjusting for unmodified DGAI score, but the results were not materially changed and are not presented. In addition, we adjusted for individual food groups instead of DGAI score, which yielded a worse model fit, and thus was not adopted for the final analyses and presentation.

The analyses of 'cream and butter' and 'fermented milk products' groups in relation to BP change and HTN risk were conducted in the same way as that for dairy foods.

\section{Results}

\section{Participants' characteristics}

Table 1 describes, by examinations, the characteristics of the participants who were included in the analysis of BP change. Blood glucose levels were higher, whereas the percentage of smokers was lower, at examination 8 compared with examination 5. Probably due to the increasing prevalence of lipidlowering medication use, there was a significant reduction in the levels of total cholesterol and fasting TAG. The HDLcholesterol level was higher at examination 8 compared with examination 5. The mean SBP exceeded $120 \mathrm{mmHg}$ from the 6th to the 8th examination, whereas mean DBP was $<75 \mathrm{mmHg}$ at all examinations. The consumption of most foods considered (including many dairy foods) was also higher at examination 8 compared with examination 5 , except for the intake of refined grains and caffeinated coffee, which tended to be lower at examination 8 . The overall diet quality (represented by the modified DGAI score) was significantly better at examination 8 compared with examination 5. In addition, as shown in online Supplementary Table S1, participants who consumed $\geq 3$ servings/d of total dairy foods also consumed higher amount of other foods (except alcohol and caffeinated coffee) than those who consumed $<1$ serving/d. They also had slightly greater BMI, but were less likely to smoke or use cholesterollowering medication.

\section{Dairy consumption and annualised change of blood pressure}

Participants who consumed $\geq 3$ servings/week of total dairy foods had 0.49 (SE 0.12$) \mathrm{mmHg}$ increment in SBP every year compared with the 1.05 (SE 0.06) $\mathrm{mmHg}$ increment among those 
Table 1. Characteristics of the participants by examinations, excluding observations with anti-hypertensive medication use during the follow-up (2075 participants with 6661 observations)‡

(Mean values and standard deviations; percentages)

\begin{tabular}{|c|c|c|c|c|c|c|c|c|}
\hline \multirow[b]{3}{*}{ Number of participants } & \multirow{2}{*}{\multicolumn{2}{|c|}{$\frac{\text { Exam } 5(1991-1995)}{1885}$}} & \multirow{2}{*}{\multicolumn{2}{|c|}{$\frac{\text { Exam } 6(1995-1998)}{1844}$}} & \multirow{2}{*}{\multicolumn{2}{|c|}{$\frac{\text { Exam } 7 \text { 1998-2001 }}{1687}$}} & \multirow{2}{*}{\multicolumn{2}{|c|}{$\frac{\text { Exam } 82005-2008}{1245}$}} \\
\hline & & & & & & & & \\
\hline & Mean & SD & Mean & SD & Mean & SD & Mean & SD \\
\hline Age (years) & 51.9 & $9 \cdot 2$ & $55 \cdot 9^{\star \star}+\dagger$ & $9 \cdot 1$ & $58 \cdot 4^{\star \star} \dagger \dagger$ & 9.0 & $63 \cdot 6^{\star \star}+\dagger$ & $8 \cdot 8$ \\
\hline Men (\%) & $42 \cdot 4$ & & $42 \cdot 6$ & & 41.9 & & 40.2 & \\
\hline BMI $\left(\mathrm{kg} / \mathrm{m}^{2}\right)$ & $26 \cdot 25$ & 4.35 & $26 \cdot 97^{\star \star}+\dagger$ & 4.74 & 27.03†† & 4.73 & $26 \cdot 88$ & 4.86 \\
\hline Regular cigarette smokers (\%) & $20 \cdot 3$ & & $16 \cdot 4^{\star \star} \dagger \dagger$ & & $14 \cdot 7^{\star \star} \dagger \dagger$ & & $10 \cdot 6^{\star *}+\dagger$ & \\
\hline Physical activity index & 34.83 & $6 \cdot 19$ & 34.87 & $6 \cdot 17$ & $37 \cdot 25+\dagger$ & $6 \cdot 18$ & 35.87 & $5 \cdot 68$ \\
\hline DGAI score & $8 \cdot 5$ & $2 \cdot 5$ & $9 \cdot 0^{\star *}+\dagger$ & $2 \cdot 6$ & $8 \cdot 7^{\star \star}$ & $2 \cdot 7$ & $9.0 \dagger$ & $2 \cdot 7$ \\
\hline Total cholesterol (mmol/l) & $5 \cdot 23$ & 0.95 & $5 \cdot 35^{\star \star}+\dagger$ & 1.07 & $5 \cdot 27^{\star *}$ & 0.94 & $5.06^{\star \star}$ & 0.93 \\
\hline HDL-cholesterol (mmol/l) & 1.35 & 0.39 & $1.37^{\star \star} \dagger \dagger$ & 0.42 & $1.46^{\star \star}+\dagger$ & 0.44 & $1.58^{\star \star}+\dagger$ & 0.46 \\
\hline TAG (mmol/l) & 1.46 & $1 \cdot 17$ & 1.49 & 1.69 & 1.40 & 0.87 & $1 \cdot 20^{\star *}+\dagger$ & 0.71 \\
\hline Glucose $(\mathrm{mmol} / \mathrm{l})$ & $5 \cdot 30$ & $1 \cdot 14$ & $5.46^{* \star}+\dagger$ & 1.14 & $5.47^{\star *}+\dagger$ & 1.09 & $5 \cdot 62^{\star \star}+\dagger$ & 1.03 \\
\hline Systolic blood pressure (mmHg) & $116 \cdot 3$ & $12 \cdot 0$ & $121 \cdot 5^{\star \star} \dagger \dagger$ & $15 \cdot 2$ & $120 \cdot 7 \dagger \dagger$ & $15 \cdot 6$ & $123 \cdot 9^{\star \star}+\dagger$ & 14.9 \\
\hline Diastolic blood pressure $(\mathrm{mmHg})$ & $71 \cdot 2$ & $8 \cdot 1$ & $73 \cdot 7^{\star \star}+\dagger$ & 8.5 & $72 \cdot 9$ & $9 \cdot 1$ & $74 \cdot 0^{\star \star}+\dagger$ & 9.2 \\
\hline Diabetics (\%) & $2 \cdot 5$ & & $4 \cdot 3^{\star \star} \dagger \dagger$ & & $4.8+\dagger$ & & 3.5 & \\
\hline Cholesterol-lowering meds users (\%) & 4.4 & & $7 \cdot 4^{\star \star}+\dagger$ & & $12 \cdot 2^{\star \star}+\dagger$ & & $26 \cdot 2^{\star \star}+\dagger$ & \\
\hline $\begin{array}{l}\text { Users of hormone-replacement therapy or oral } \\
\text { contraceptives (\%) }\end{array}$ & 13.5 & & $20^{\star \star}+\dagger$ & & $22 \cdot 8^{*}+\dagger$ & & $\mathrm{N} / \mathrm{A}$ & \\
\hline \multicolumn{9}{|l|}{ Dietary intake } \\
\hline Total energy (kJ) & 7833.8 & $2575 \cdot 9$ & $7830 \cdot 3$ & $2592 \cdot 2$ & 7738.9 & $2536 \cdot 6$ & 7911.9 & $2666 \cdot 3$ \\
\hline Fish (g/week) & 230.0 & 191.8 & $217 \cdot 5^{\star \star} \dagger \dagger$ & $177 \cdot 3$ & $228 \cdot 3$ & 197.9 & 239.8 & 219.5 \\
\hline Meat (g/week) & $868 \cdot 6$ & 476.9 & 841.9 & $445 \cdot 6$ & $852 \cdot 1$ & $440 \cdot 0$ & 887.9 & $457 \cdot 6$ \\
\hline Whole grain (g/week) & $397 \cdot 8$ & 455.9 & $427 \cdot 3^{*} \dagger$ & $495 \cdot 8$ & $436 \cdot 3$ & 471.4 & $570 \cdot 0^{\star \star} \dagger \dagger$ & $513 \cdot 0$ \\
\hline Refined grain (g/week) & 568.5 & $380 \cdot 2$ & $597 \cdot 8$ & $486 \cdot 7$ & $514 \cdot 9^{\star \star}+\dagger$ & $366 \cdot 9$ & $384 \cdot 3^{\star \star}+\dagger$ & 363.0 \\
\hline Fruits and vegetables (g/week) & 3550.0 & $2064 \cdot 0$ & $3644 \cdot 2$ & $1949 \cdot 2$ & $3722.4 \dagger \dagger$ & $2037 \cdot 1$ & $3541 \cdot 0^{\star \star}$ & $1932 \cdot 3$ \\
\hline Alcohol (g/week) & 1030.6 & $2057 \cdot 3$ & 1037.9 & 1979.2 & 980.3 & $1782 \cdot 2$ & $928 \cdot 8$ & $1747 \cdot 7$ \\
\hline Caffeinated coffee (g/week) & $3081 \cdot 1$ & $2702 \cdot 2$ & $2804 \cdot 3^{\star *} \dagger \dagger$ & $2499 \cdot 8$ & $2553 \cdot 4^{\star \star}+\dagger$ & $2355 \cdot 2$ & $2281 \cdot 9 \dagger \dagger$ & $2144 \cdot 2$ \\
\hline \multicolumn{9}{|l|}{ Dairy and milk products } \\
\hline Total dairy products (servings/d) & 1.49 & $1 \cdot 10$ & 1.53 & 1.17 & 1.58 & 1.19 & $1 \cdot 70^{\star \star}+\dagger$ & 1.22 \\
\hline High-fat dairy products (servings/week) & 4.48 & 4.58 & $4 \cdot 35$ & 4.77 & $4 \cdot 83^{\star *}$ & $5 \cdot 30$ & $5 \cdot 66^{\star *}+\dagger$ & $5 \cdot 51$ \\
\hline Low-fat dairy products (servings/week) & 5.95 & $6 \cdot 43$ & $6 \cdot 35$ & $6 \cdot 80$ & $6 \cdot 22$ & $6 \cdot 84$ & $6 \cdot 28$ & 6.74 \\
\hline Cheese (servings/week) & 2.74 & 2.94 & $2 \cdot 71$ & $3 \cdot 16$ & $3.05^{\star}$ & 3.71 & $3.73^{\star *}+\dagger$ & $4 \cdot 2$ \\
\hline Yoghurt (servings/week) & 0.91 & 1.96 & $1 \cdot 14^{\star \star}+\dagger$ & $2 \cdot 28$ & $1 \cdot 14 \dagger \dagger$ & 2.43 & $1.68^{\star *}+\dagger$ & 3.46 \\
\hline Skimmed/low-fat milk (servings/week) & 4.70 & $5 \cdot 88$ & 4.86 & 6.09 & 4.77 & 5.89 & $4 \cdot 30$ & 5.37 \\
\hline Fluid milk (servings/week) & 5.43 & $6 \cdot 12$ & $5 \cdot 44$ & $6 \cdot 4$ & $5 \cdot 33$ & $6 \cdot 18$ & $4.81 \dagger \dagger$ & $5 \cdot 68$ \\
\hline Whole milk (servings/week) & 0.73 & 2.52 & $0.58^{* *}+\dagger$ & $2 \cdot 29$ & 0.56 & $2 \cdot 62$ & $0.51+\dagger$ & $2 \cdot 29$ \\
\hline Fermented milk products (servings/week) & 4.30 & 3.89 & 4.66 & 4.42 & $4.94 \uparrow \dagger$ & 4.87 & $6 \cdot 11^{\star *}+\dagger$ & 5.74 \\
\hline Cream and butter (servings/week) & $5 \cdot 67$ & 9.42 & $5 \cdot 76^{\star \star}+\dagger$ & 8.6 & $6.08+\dagger$ & $8 \cdot 73$ & $6 \cdot 28+\dagger$ & $9 \cdot 17$ \\
\hline
\end{tabular}

DGAI, Dietary Guidelines Adherence Index.

Mean value was significantly different from that for the previous examination: ${ }^{*} P<0.05,{ }^{*} P<0.01$

Mean value was significantly different from that for examination $5: \dagger P<0.05$, $\uparrow+P<0.01$

¥ Comparisons between groups were tested, with Bonferroni correction by paired $t$ test or sign rank test for continuous variables or by $x^{2}$ test for categorical variables. Total dairy products included skimmed/low-fat milk, whole milk, sherbet/ice milk, ice cream, yoghurt, cottage/ricotta cheese and other cheese (e.g. American, Cheddar, etc.). Low-fat dairy products included skimmed/low-fat milk, sherbet/ice milk and yoghurt. High-fat dairy products included whole milk, ice cream, cottage/ricotta cheese and other cheese (e.g. American, Cheddar, etc.). Fluid milk include skimmed/low-fat milk and whole milk. According to the definition by the US Department of Agriculture MyPlate Dairy Group ${ }^{(28)}$ and the Dietary Guidelines for Americans ${ }^{(29)}$, milk products with little to no Ca (e.g. cream cheese, cream and butter) are not included in the Dairy Group. Therefore, for the present study, we did not include cream, sour cream, cream cheese and butter in any of the dairy groups. However, for the purpose of comparison, we created a separate group 'Cream and butter' by summing the intake of these four milk products. In addition, the 'Fermented milk products' group includes sour cream, yoghurt, cottage/ricotta cheese, cream cheese and other cheese (e.g. American, Cheddar, etc.). For all dairy/milk products, we used unit 'servings' to facilitate the comparisons across different diary/milk products. The conversion algorithm between 'serving' and 'gram' is 1 serving skimmed/low-fat milk $=245 \mathrm{~g} ; 1$ serving whole milk $=244 \mathrm{~g} ; 1$ serving cream $=15 \mathrm{~g} ; 1 \mathrm{serving}$ sour cream $=12 \mathrm{~g}$; 1 serving sherbet/ice milk = 96 g; 1 serving ice cream =66 g; 1 serving yoghurt = $227 \mathrm{~g} ; 1$ serving cottage/ricotta cheese = $105 \mathrm{~g} ; 1$ serving cream cheese $=28 \mathrm{~g} ; 1$ serving other cheese (e.g. American, Cheddar, etc.) $=28 \mathrm{~g} ; 1$ serving butter $=5 \mathrm{~g}$.

who consumed $<1$ serving/week $\left(P_{\text {trend }}<0 \cdot 001\right)$, adjusting for demographic and lifestyle factors (Table 2). An inverse association was also evident between total dairy consumption and the annualised change in DBP $\left(P_{\text {trend }}=0.005\right.$; Table 3$)$. Similar significant (all $P_{\text {trend }}<0.05$ ) inverse associations with the annualised change in SBP were also observed for the consumption of total low-fat dairy products, fluid milk, skimmed/ low-fat milk, yogurt and fermented milk products. However, the intakes of skimmed/low-fat milk, yoghurt and fermented milk products were not associated with the change in DBP.
The consumption of total high-fat dairy products, whole milk, cheese or cream and butter was not significantly associated with the annualised change in either SBP or DBP.

The above findings were not materially changed (1) with further adjustment for time-varying BMI (model 3 in both Tables 2 and 3); (2) with additional adjustment for the use of cholesterol-lowering medication, hormone-replacement therapy or oral contraceptives (in women), diabetic status and lipid profiles (data not shown); or (3) in the sensitivity analysis of BP change that included an additional 1066 observations with 
Table 2. Annualised change of systolic blood pressure across groups of dairy/milk-product consumption, excluding observations with anti-hypertensive medication use during the follow-up (2075 participants with 6661 observations)

(Mean values with their standard errors; medians and ranges)

\begin{tabular}{|c|c|c|c|c|c|c|c|}
\hline & \multicolumn{6}{|c|}{ Groups of dairy consumption } & \multirow[b]{3}{*}{$P_{\text {trend }}$} \\
\hline & \multicolumn{2}{|c|}{$<1$ servings } & \multicolumn{2}{|c|}{1 to $<3$ servings } & \multicolumn{2}{|c|}{$\geq 3$ servings } & \\
\hline & Mean* & SE & Mean & SE & Mean & SE & \\
\hline \multicolumn{8}{|c|}{ Total dairy consumption (servings/d) } \\
\hline Median & \multicolumn{2}{|c|}{$0.639 \dagger$} & \multicolumn{2}{|c|}{1.636} & \multicolumn{2}{|c|}{3.525} & \\
\hline Range & \multicolumn{2}{|c|}{$0,0.998$} & \multicolumn{2}{|c|}{$1.000,2.998$} & \multicolumn{2}{|c|}{$3.000,7.531$} & \\
\hline$n$ & & & & & & & \\
\hline Model 1‡ & 1.07 & 0.06 & 0.78 & 0.05 & 0.51 & 0.12 & $<0.001$ \\
\hline Model $2 \S$ & 1.05 & 0.06 & 0.78 & 0.05 & 0.49 & 0.12 & $<0.001$ \\
\hline Model 3\| & 1.07 & 0.06 & 0.77 & 0.05 & 0.47 & 0.12 & $<0.001$ \\
\hline Total low-fat/ & y consur & /ings/u & & & & & \\
\hline Median & & & & & & & \\
\hline Range & & & & & & & \\
\hline$n$ & & & & & & & \\
\hline Model 1 & $1 \cdot 12$ & 0.08 & 0.92 & 0.09 & 0.75 & 0.04 & $<0.001$ \\
\hline Model 2 & $1 \cdot 10$ & 0.08 & 0.90 & 0.09 & 0.75 & 0.04 & $<0.001$ \\
\hline Model 3 & $1 \cdot 12$ & 0.08 & 0.90 & 0.09 & 0.75 & 0.04 & $<0.001$ \\
\hline Total high-fat & umption & eek) & & & & & \\
\hline Median & & & & & & & \\
\hline Range & & & & & & & \\
\hline$n$ & & & & & & & \\
\hline Model 1 & 0.91 & 0.11 & 0.79 & 0.07 & 0.87 & 0.05 & 0.69 \\
\hline Model 2 & 0.94 & 0.12 & 0.79 & 0.07 & 0.85 & 0.05 & 0.92 \\
\hline Model 3 & 0.95 & 0.12 & 0.79 & 0.07 & 0.85 & 0.05 & 0.95 \\
\hline Fluid milk (se & & & & & & & \\
\hline Median & & & & & & & \\
\hline Range & & & & & & & \\
\hline$n$ & & & & & & & \\
\hline Model 1 & 1.07 & 0.07 & 0.89 & 0.09 & 0.76 & 0.04 & $<0.001$ \\
\hline Model 2 & 1.05 & 0.08 & 0.88 & 0.09 & 0.76 & 0.04 & 0.001 \\
\hline Model 3 & 1.07 & 0.08 & 0.87 & 0.09 & 0.75 & 0.05 & $<0.001$ \\
\hline Whole milk & ek) & & & & & & \\
\hline Median & & & & & & & \\
\hline Range & & & & & & & \\
\hline$n$ & & & & & & & \\
\hline Model 1 & 0.85 & 0.04 & 0.8 & $0 \cdot 16$ & 0.88 & 0.14 & 0.9245 \\
\hline Model 2 & 0.85 & 0.04 & 0.78 & $0 \cdot 16$ & 0.83 & 0.14 & 0.8293 \\
\hline Model 3 & 0.85 & 0.04 & 0.79 & $0 \cdot 16$ & 0.82 & 0.14 & 0.7829 \\
\hline Skimmed/low & ervings/v & & & & & & \\
\hline Median & & & & & & & \\
\hline Range & & & & & & & \\
\hline$n$ & & & & & & & \\
\hline Model 1 & 1.04 & 0.06 & 0.89 & 0.09 & 0.74 & 0.05 & $<0.001$ \\
\hline Model 2 & 1.02 & 0.07 & 0.87 & 0.09 & 0.74 & 0.05 & $<0.001$ \\
\hline Model 3 & 1.04 & 0.07 & 0.87 & 0.09 & 0.73 & 0.05 & $<0.001$ \\
\hline Cheese (ser & & & & & & & \\
\hline Median & & & & & & & \\
\hline Range & & & & & & & \\
\hline$n$ & & & & & & & \\
\hline Model 1 & 0.92 & 0.08 & 0.82 & 0.06 & 0.84 & 0.05 & 0.57 \\
\hline Model 2 & 0.91 & 0.08 & 0.81 & 0.06 & 0.83 & 0.06 & 0.52 \\
\hline Model 3 & 0.92 & 0.08 & 0.81 & 0.06 & 0.83 & 0.06 & 0.48 \\
\hline Yoghurt (serv & & & & & & & \\
\hline Median & & & & & & & \\
\hline Range & & & & & & & \\
\hline$n$ & & & & & & & \\
\hline Model 1 & 0.92 & 0.04 & 0.71 & 0.08 & 0.71 & 0.09 & 0.01 \\
\hline Model 2 & 0.90 & 0.04 & 0.73 & 0.08 & 0.72 & 0.09 & 0.04 \\
\hline Model 3 & 0.91 & 0.04 & 0.72 & 0.08 & 0.71 & 0.09 & 0.03 \\
\hline Cream and b & ngs/weel & & & & & & \\
\hline Median & & & & & & & \\
\hline Range & & & & & & & \\
\hline$n$ & & & & & & & \\
\hline Model 1 & 0.79 & 0.06 & 0.88 & 0.08 & 0.88 & 0.05 & 0.40 \\
\hline Model 2 & 0.80 & 0.07 & 0.89 & 0.08 & 0.85 & 0.05 & 0.81 \\
\hline Model 3 & 0.80 & 0.07 & 0.87 & 0.08 & 0.85 & 0.05 & 0.73 \\
\hline
\end{tabular}


Table 2 Continued

\begin{tabular}{|c|c|c|c|c|c|c|c|}
\hline & \multicolumn{6}{|c|}{ Groups of dairy consumption } & \multirow[b]{3}{*}{$P_{\text {trend }}$} \\
\hline & \multicolumn{2}{|c|}{$<1$ servings } & \multicolumn{2}{|c|}{1 to $<3$ servings } & \multicolumn{2}{|c|}{$\geq 3$ servings } & \\
\hline & Mean* $^{*}$ & SE & Mean & SE & Mean & SE & \\
\hline \multicolumn{8}{|c|}{ Fermented milk products (servings/week) } \\
\hline Median & \multicolumn{2}{|c|}{0.705} & \multicolumn{2}{|c|}{2.000} & \multirow{2}{*}{\multicolumn{2}{|c|}{$\begin{array}{c}5.690 \\
3.000,34.940\end{array}$}} & \\
\hline Range & \multirow{2}{*}{\multicolumn{2}{|c|}{$\begin{array}{c}0,0.970 \\
484\end{array}$}} & \multirow{2}{*}{\multicolumn{2}{|c|}{$\begin{array}{c}1.000,2.985 \\
1166\end{array}$}} & & & \\
\hline$n$ & & & & & \multicolumn{2}{|c|}{2936} & \\
\hline Model 1 & 1.08 & 0.12 & 0.92 & 0.07 & 0.78 & 0.04 & 0.01 \\
\hline Model 2 & 1.06 & 0.12 & 0.90 & 0.07 & 0.78 & 0.05 & 0.03 \\
\hline Model 3 & 1.07 & 0.12 & 0.90 & 0.07 & 0.78 & 0.05 & 0.02 \\
\hline
\end{tabular}

$n$, Number of observations of the change in systolic blood pressure.

* Mean annualised change in systolic blood pressure $(\mathrm{mmHg})$ for all such values.

$\dagger$ Total dairy products included skimmed/low-fat milk, whole milk, sherbet/ice milk, ice cream, yoghurt, cottage/ricotta cheese and other cheese (e.g. American, Cheddar, etc.). Lowfat dairy products included skimmed/low-fat milk, sherbet/ice milk and yoghurt. High-fat dairy products included whole milk, ice cream, cottage/ricotta cheese and other cheese (e.g. American, Cheddar, etc.). Fluid milk includes skimmed/low-fat milk and whole milk. According to the definition by the US Department of Agriculture MyPlate Dairy Group $^{(28)}$ and the Dietary Guidelines for Americans ${ }^{(29)}$, milk products with little to no Ca (e.g. cream cheese, cream and butter) are not included in the Dairy Group. Therefore, for the present study, we did not include cream, sour cream, cream cheese and butter in any of the dairy groups. However, for the purpose of comparison, we created a separate group 'Cream and butter' by summing the intake of these four milk products. In addition, the 'Fermented milk products' group included sour cream, yoghurt, cottage/ricotta cheese, cream cheese and other cheese (e.g. American, Cheddar, etc.). For all dairy/milk products, we used unit 'servings' to facilitate the comparisons across different diary/milk products. The conversion algorithm between 'serving' and 'gram' is 1 serving skimmed/low-fat milk = $245 \mathrm{~g} ; 1$ serving whole milk=244 g; 1 serving cream = $15 \mathrm{~g}$; 1 serving sour cream $=12 \mathrm{~g} ; 1$ serving sherbet/ice milk $=96 \mathrm{~g} ; 1$ serving ice cream $=66 \mathrm{~g} ; 1$ serving yoghurt $=227 \mathrm{~g} ; 1$ serving cottage/ricotta cheese $=105 \mathrm{~g} ; 1$ serving cream cheese $=28 \mathrm{~g}$; 1 serving other cheese (e.g. American, Cheddar, etc.) $=28 \mathrm{~g}$; and 1 serving butter $=5 \mathrm{~g}$.

¥ Model 1: adjusted for sex, age and systolic blood pressure at the beginning of each exam interval and average total energy intake during each exam interval.

$\S$ Model 2: model $1+$ smoking status and physical activity at the beginning of each exam interval and the average caffeine coffee intake and Dietary Guidelines Adherence Index (DGAI) sub-score (i.e. DGAI score excluding sub-scores for assessing the consumption amount of milk and milk products and the likelihood of choosing low-fat milk and milk products) during each exam interval.

|| Model 3: model 2+BMl at the beginning of each exam interval.

anti-hypertensive medication use during follow-up (online Supplementary Tables S2 and S3).

\section{Dairy consumption and risk of incident hypertension}

During a median 14.6-year follow-up, 1026 out of 2340 participants developed incident HTN. The proportional hazards assumption was met for most of the dairy and fermented milk products, except for skimmed/low-fat milk, total low-fat dairy products and cream and butter. After adjusting for demographic and lifestyle factors (Table 4, model 2), consuming 1 more serving/week of yoghurt was related to 6 (95\% CI 1, 10) \% reduced risk of developing incident HTN $(P=0 \cdot 01)$. Similar inverse association was also found between the risk of incident HTN and the consumption of total dairy $(P=0.04)$ and fermented milk products $(P=0 \cdot 01)$. In contrast with the finding on annualised BP change, we found a weak but significantly inverse association between the consumption of total high-fat dairy intake and the risk of incident HTN ( $\beta=-0.030 ; P=0.002$ per 1 serving/week increment in total high-fat dairy intake).

The violation of proportional hazards assumption indicates that the relative risk of incident HTN between people with high $v$. low skimmed/low-fat milk consumption was not constant with time. We observed a significant inverse association between the consumption of skimmed/low-fat milk and the HTN incidence earlier during follow-up. However, the positive interaction $(P=0.01)$ between skimmed/low-fat milk intake and follow-up time indicated that later during follow-up the inverse association was attenuated (Table 4). Similar inverse associations with attenuation were observed for total low-fat/fat-free dairy products (most likely driven by skimmed/low-fat milk), as well as cream and butter. To better demonstrate this interaction, we stratified follow-up time as $<8$ and $\geq 8$ years and re-ran these analyses. For follow-up $<8$ years, each additional serving/week of skimmed/low-fat milk intake was associated with a $2 \%$ (HR 0.98; 95\% CI 0.97, 0.999; $P=0.04$ ) lower risk of incident HTN. For follow-up $\geq 8$ years, skimmed/low-fat milk intake was unrelated to the incidence of HTN (HR 1.02; $95 \%$ CI 0.995, 1.04; $P=0 \cdot 14$ ).

These findings were not changed when further adjusting for time-varying BMI (Table 4) or other factors such as hormonereplacement therapy or oral contraceptives (in women) (data not shown) or in the sensitivity analysis that excluded 130 participants who did not come back after their baseline examinations (online Supplementary Table S4).

\section{Discussion}

Among the FHS Offspring Cohort of middle-aged to older adults, greater consumption ( $\geq 3$ servings/d or week $v .<1$ serving) of total dairy, yoghurt, skimmed/low-fat milk and fermented milk products was associated with a $0 \cdot 2-0 \cdot 6 \mathrm{mmHg}$ smaller increment in SBP/year. Similar inverse associations with SBP change were also observed for total low-fat/fat-free dairy foods and fluid milk, which was possibly driven by yoghurt and skimmed/low-fat milk. In addition, the consumption of total dairy, total low-fat/fat-free dairy products and fluid milk was also inversely related to annualised DBP change. Consistent with the findings on SBP change, greater total dairy foods and yoghurt intake was found to be associated with a lower HTN incidence, with a constant hazards ratio over the average 
Table 3. Annualised change of diastolic blood pressure across groups of dairy/milk-product consumption, excluding observations with anti-hypertensive medication use during the follow-up (2075 participants with 6661 observations)

(Mean values with their standard errors; medians and ranges)

\begin{tabular}{|c|c|c|c|c|c|c|c|}
\hline & \multicolumn{6}{|c|}{ Groups of dairy consumption } & \multirow[b]{3}{*}{$P_{\text {trend }}$} \\
\hline & \multicolumn{2}{|c|}{$<1$ servings } & \multicolumn{2}{|c|}{1 to $<3$ servings } & \multicolumn{2}{|c|}{$\geq 3$ servings } & \\
\hline & Mean* & SE & Mean & SE & Mean & SE & \\
\hline \multicolumn{8}{|c|}{ Total dairy consumption (servings/d) } \\
\hline Median & \multicolumn{2}{|c|}{0.639} & \multicolumn{2}{|c|}{1.636} & \multicolumn{2}{|c|}{3.525} & \\
\hline Range & \multicolumn{2}{|c|}{$0,0.998 \dagger$} & \multicolumn{2}{|c|}{$1.000,2.998$} & \multicolumn{2}{|c|}{$3.000,7.531$} & \\
\hline$n$ & & & & & & & \\
\hline Model 1‡ & 0.42 & 0.04 & 0.30 & 0.03 & 0.20 & 0.07 & 0.008 \\
\hline Model $2 \S$ & 0.41 & 0.04 & 0.30 & 0.03 & 0.17 & 0.07 & 0.005 \\
\hline Model 3\| & 0.41 & 0.04 & 0.30 & 0.03 & 0.17 & 0.08 & 0.004 \\
\hline Total low-fat/ & y consun & ings/w & & & & & \\
\hline Median & & & & & & & \\
\hline Range & & & & & & & \\
\hline$n$ & & & & & & & \\
\hline Model 1 & 0.42 & 0.05 & 0.39 & 0.06 & 0.29 & 0.03 & 0.01 \\
\hline Model 2 & 0.42 & 0.05 & 0.37 & 0.06 & 0.28 & 0.03 & 0.02 \\
\hline Model 3 & 0.42 & 0.05 & 0.38 & 0.06 & 0.28 & 0.03 & 0.01 \\
\hline Total high-fat & umption & eek) & & & & & \\
\hline Median & & & & & & & \\
\hline Range & & & & & & & \\
\hline$n$ & & & & & & & \\
\hline Model 1 & 0.24 & 0.07 & 0.3 & 0.04 & 0.36 & 0.03 & 0.12 \\
\hline Model 2 & 0.25 & 0.07 & 0.3 & 0.04 & 0.35 & 0.03 & 0.20 \\
\hline Model 3 & 0.25 & 0.07 & 0.3 & 0.04 & 0.35 & 0.03 & 0.22 \\
\hline Fluid milk (se & & & & & & & \\
\hline Median & & & & & & & \\
\hline Range & & & & & & & \\
\hline$n$ & & & & & & & \\
\hline Model 1 & 0.41 & 0.05 & 0.39 & 0.06 & 0.29 & 0.03 & 0.02 \\
\hline Model 2 & 0.39 & 0.05 & 0.37 & 0.06 & 0.28 & 0.03 & 0.03 \\
\hline Model 3 & 0.4 & 0.05 & 0.38 & 0.06 & 0.28 & 0.03 & 0.03 \\
\hline Whole milk ( & ek) & & & & & & \\
\hline Median & & & & & & & \\
\hline Range & & & & & & & \\
\hline$n$ & & & & & & & \\
\hline Model 1 & 0.34 & 0.02 & 0.41 & 0.1 & 0.15 & 0.09 & 0.0933 \\
\hline Model 2 & 0.33 & 0.02 & 0.4 & 0.1 & 0.16 & 0.09 & 0.1397 \\
\hline Model 3 & 0.33 & 0.02 & 0.39 & 0.1 & 0.16 & 0.09 & 0.1163 \\
\hline Skimmed/low & ervings/W & & & & & & \\
\hline Median & & & & & & & \\
\hline Range & & & & & & & \\
\hline$n$ & & & & & & & \\
\hline Model 1 & 0.38 & 0.04 & 0.35 & 0.06 & 0.29 & 0.03 & 0.08 \\
\hline Model 2 & 0.38 & 0.04 & 0.35 & 0.06 & 0.29 & 0.03 & $0 \cdot 10$ \\
\hline Model 3 & 0.38 & 0.04 & 0.35 & 0.06 & 0.29 & 0.03 & 0.09 \\
\hline Cheese (ser & & & & & & & \\
\hline Median & & & & & & & \\
\hline Range & & & & & & & \\
\hline$n$ & & & & & & & \\
\hline Model 1 & 0.29 & 0.05 & 0.32 & 0.04 & 0.35 & 0.04 & 0.36 \\
\hline Model 2 & 0.29 & 0.05 & 0.32 & 0.04 & 0.34 & 0.04 & 0.44 \\
\hline Model 3 & 0.29 & 0.05 & 0.32 & 0.04 & 0.34 & 0.04 & 0.44 \\
\hline Yoghurt (serv & & & & & & & \\
\hline Median & & & & & & & \\
\hline Range & & & & & & & \\
\hline$n$ & & & & & & & \\
\hline Model 1 & 0.36 & 0.03 & 0.29 & 0.05 & 0.24 & 0.06 & 0.05 \\
\hline Model 2 & 0.35 & 0.03 & 0.29 & 0.05 & 0.25 & 0.06 & 0.10 \\
\hline Model 3 & 0.35 & 0.03 & 0.29 & 0.05 & 0.24 & 0.06 & 0.09 \\
\hline Cream and b & ngs/week & & & & & & \\
\hline Median & & & & & & & \\
\hline Range & & & & & & & \\
\hline$n$ & & & & & & & \\
\hline Model 1 & 0.26 & 0.04 & 0.34 & 0.05 & 0.37 & 0.03 & 0.07 \\
\hline Model 2 & 0.27 & 0.04 & 0.34 & 0.05 & 0.35 & 0.03 & 0.24 \\
\hline Model 3 & 0.27 & 0.04 & 0.34 & 0.05 & 0.35 & 0.03 & 0.22 \\
\hline
\end{tabular}


Table 3 Continued

\begin{tabular}{|c|c|c|c|c|c|c|c|}
\hline & \multicolumn{6}{|c|}{ Groups of dairy consumption } & \multirow[b]{3}{*}{$P_{\text {trend }}$} \\
\hline & \multicolumn{2}{|c|}{$<1$ servings } & \multicolumn{2}{|c|}{1 to $<3$ servings } & \multicolumn{2}{|c|}{$\geq 3$ servings } & \\
\hline & Mean* & $\mathrm{SE}$ & Mean & $\mathrm{SE}$ & Mean & SE & \\
\hline \multicolumn{8}{|c|}{ Fermented milk products (servings/week) } \\
\hline Median & \multirow{2}{*}{\multicolumn{2}{|c|}{$\begin{array}{c}0.705 \\
0,0.970\end{array}$}} & \multirow{2}{*}{\multicolumn{2}{|c|}{$\begin{array}{c}2.000 \\
1.000,2.985\end{array}$}} & \multicolumn{2}{|c|}{5.690} & \\
\hline Range & & & & & \multirow{2}{*}{\multicolumn{2}{|c|}{$\begin{array}{c}3.000,34.940 \\
2936\end{array}$}} & \\
\hline$n$ & \multicolumn{2}{|c|}{484} & \multicolumn{2}{|c|}{1166} & & & \\
\hline Model 1 & 0.31 & 0.07 & 0.32 & 0.05 & 0.34 & 0.03 & 0.62 \\
\hline Model 2 & 0.29 & 0.07 & 0.31 & 0.05 & 0.33 & 0.03 & 0.62 \\
\hline Model 3 & 0.29 & 0.07 & 0.31 & 0.05 & 0.33 & 0.03 & 0.61 \\
\hline
\end{tabular}

$n$, Number of observations of the change in systolic blood pressure.

* Mean annualised change of diastolic blood pressure $(\mathrm{mmHg})$ for all such values.

$\dagger$ Total dairy products included skimmed/low-fat milk, whole milk, sherbet/ice milk, ice cream, yoghurt, cottage/ricotta cheese and other cheese (e.g. American, Cheddar, etc.). Lowfat dairy products included skimmed/low-fat milk, sherbet/ice milk and yoghurt. High-fat dairy products included whole milk, ice cream, cottage/ricotta cheese and other cheese (e.g. American, Cheddar, etc.). Fluid milk includes skimmed/low-fat milk and whole milk. According to the definition by the US Department of Agriculture MyPlate Dairy Group $^{(28)}$ and the Dietary Guidelines for Americans ${ }^{(29)}$, milk products with little to no Ca (e.g. cream cheese, cream and butter) are not included in the Dairy Group. Therefore, for the present study, we did not include cream, sour cream, cream cheese and butter in any of the dairy groups. However, for the purpose of comparison, we created a separate group 'Cream and butter' by summing the intake of these four milk products. In addition, the 'Fermented milk products' group includes sour cream, yoghurt, cottage/ricotta cheese, cream cheese and other cheese (e.g. American, Cheddar, etc.). For all dairy/milk products, we used unit 'servings' to facilitate the comparisons across different diary/milk products. The conversion algorithm between 'serving' and 'gram' is 1 serving skimmed/low-fat milk = $245 \mathrm{~g} ; 1$ serving whole milk=244 g; 1 serving cream = $15 \mathrm{~g}$; 1 serving sour cream $=12 \mathrm{~g} ; 1$ serving sherbet/ice milk $=96 \mathrm{~g} ; 1$ serving ice cream $=66 \mathrm{~g} ; 1$ serving yoghurt $=227 \mathrm{~g} ; 1$ serving cottage/ricotta cheese = $105 \mathrm{~g} ; 1$ serving cream cheese $=28 \mathrm{~g}$; 1 serving other cheese (e.g. American, Cheddar, etc.) $=28 \mathrm{~g} ; 1$ serving butter $=5 \mathrm{~g}$.

¥ Model 1: adjusted for sex, age and diastolic blood pressure at the beginning of each exam interval and average total energy intake during each exam interval.

$\S$ Model 2: model $1+$ smoking status and physical activity at the beginning of each exam interval and the average caffeine coffee intake and Dietary Guidelines Adherence Index (DGAI) sub-score (i.e. DGAI score excluding sub-scores for assessing the consumption amount of milk and milk products and the likelihood of choosing low-fat milk and milk products) during each exam interval.

|| Model 3: model 2+BMl at the beginning of each exam interval.

15-year follow-up. In contrast, although higher intakes of skimmed/low-fat milk were associated with a lower HTN incidence earlier during the follow-up period (i.e. each additional serving/week of skimmed/low-fat milk intake was significantly associated with a $2 \%$ lower risk of incident HTN), the magnitude of this association seemed to diminish over time (i.e. as individuals get older). A similar pattern was observed for the total low-fat dairy group (likely driven by skimmed/low-fat milk). Based on the slowed, but continued increase in SBP, with higher dairy intake, these findings suggest that skimmed/low-fat milk may delay the onset, but not lower the eventual risk, of HTN as individuals get older - that is, it may slow down, but not stop, the rise in SBP with age - and delay the development of HTN with ageing but not prevent HTN at older ages. Future studies are warranted to confirm our findings and explore the underlying mechanisms in this regard.

Poor diet is one of the risk factors for elevated $\mathrm{BP}$ and the development of $\mathrm{HTN}^{(31)}$. Although accumulating evidence suggests that greater dairy intake, as a critical component of a healthy dietary pattern, is related to lower BP among adults $^{(10,29)}$, the findings on different types of dairy foods in this regard varied across studies ${ }^{(12)}$. Among the longitudinal studies that have examined a variety of dairy products, Heraclides et $a l .^{(21)}$ observed no favourable change in BP or HTN risk in 1750 participants with the consumption (assessed once by 5-d food diaries at baseline) of dairy products, including total dairy (with or without cheese, median intake about 2 servings/d in the highest tertile group), low-fat dairy, full-fat dairy and fermented dairy intakes after 10 years of follow-up. In contrast, with a 5-year follow-up (dairy intake assessed once by FFQ at baseline with median total dairy intake about $2 \cdot 3$ servings/d),
Engberink et al ${ }^{(37)}$ reported lower HTN risk with higher low-fat dairy intake in 3454 Dutch participants and lower BP with more total milk products consumed in 21553 participants; total fermented dairy intake was only inversely associated with DBP, but not with SBP, whereas neither cheese nor yoghurt were individually related to $\mathrm{BP}$ or HTN risk. The diverse population characteristics and study methodologies (e.g. follow-up duration, sample size) may partially explain the study discrepancies.

Dairy foods are rich sources of various bioactive peptides, medium-chain fatty acids and micronutrients such as $\mathrm{Ca}, \mathrm{K}$ and $\mathrm{Mg}$, which are among the nutrients that are currently underconsumed by adult Americans ${ }^{(38)}$. These nutrients may contribute to potential underlying mechanisms for the BP-lowering effect of dairy products ${ }^{(12,39,40)}$, such as regulating vascular resistance and promoting vasodilation by enhancing the synthesis of nitric oxide, reducing renal $\mathrm{Na}$ retention, blocking $\mathrm{Ca}^{2+}$ channels and reducing intracellular $\mathrm{Ca}^{2+(9-13)}$.

Ageing is known to be a strong determinant of rising SBP and isolated systolic $\mathrm{HTN}^{(41)}$. Blood vessels become less distensible with ageing, which induces isolated SBP augmentation and can be worsened by excessive drug-induced DBP reduction ${ }^{(42)}$. Recent studies have found that greater total dairy consumption predicted reduced arterial stiffness (as measured by pulse wave velocity or augmentation index) ${ }^{(43,44)}$, and milk consumption is associated with lower $\mathrm{SBP}^{(43)}$. Our findings suggest that skimmed/low-fat milk may help slow down the rise in SBP with age and consequently delay, although not prevent, the onset of systolic HTN. In contrast, the relation between lower risk of incident HTN during follow-up with greater consumption of yoghurt was maintained throughout follow-up. It has been 
Table 4. Hazard ratios (HR) of incident hypertension with each 1 serving/d per week increasing consumption of dairy and milk products (2340 participants) ( $\beta$ Coefficients, hazard ratios and $95 \%$ confidence intervals)

\begin{tabular}{|c|c|c|c|c|c|c|c|c|c|c|c|c|}
\hline \multirow[b]{3}{*}{ Total dairy products (serving/d) } & \multicolumn{4}{|c|}{ Model $1 \dagger$} & \multicolumn{4}{|c|}{ Model $2 \ddagger$} & \multicolumn{4}{|c|}{ Model $3 \S$} \\
\hline & \multirow{2}{*}{$\frac{\beta}{-0.084}$} & \multicolumn{2}{|c|}{$\mathrm{HR}(95 \% \mathrm{Cl})$} & \multirow{2}{*}{$\frac{P}{0.02}$} & \multirow{2}{*}{$\frac{\beta}{-0.077}$} & \multicolumn{2}{|c|}{$\mathrm{HR}(95 \% \mathrm{Cl})$} & \multirow{2}{*}{$\frac{P}{0.04}$} & \multirow{2}{*}{$\frac{\beta}{-0.084}$} & \multicolumn{2}{|c|}{$\mathrm{HR}(95 \% \mathrm{Cl})$} & \multirow{2}{*}{$\frac{P}{0.02}$} \\
\hline & & 0.92 & $0.86,0.99$ & & & 0.93 & $0.86,0.996$ & & & 0.92 & $0.86,0.99$ & \\
\hline Total high-fat dairy products (serving/week) & -0.022 & 0.98 & $0.96,0.996$ & 0.02 & -0.030 & 0.97 & $0.95,0.99$ & 0.002 & -0.030 & 0.97 & $0.95,0.99$ & 0.002 \\
\hline Fluid milk (serving/week) & -0.005 & 1.00 & $0.98,1.01$ & 0.39 & -0.004 & 1.00 & $0.98,1.01$ & 0.53 & -0.005 & 1.00 & $0.98,1.01$ & 0.45 \\
\hline Whole milk (serving/week) & -0.012 & 0.99 & $0.96,1.02$ & 0.43 & -0.024 & 0.98 & $0.95,1.01$ & 0.15 & -0.017 & 0.98 & $0.95,1.02$ & 0.30 \\
\hline Cheese (serving/week) & -0.017 & 0.98 & $0.96,1.01$ & 0.20 & -0.020 & 0.98 & $0.95,1.01$ & 0.15 & -0.022 & 0.98 & $0.95,1.01$ & 0.11 \\
\hline Yoghurt (serving/week) & -0.068 & 0.93 & $0.89,0.98$ & 0.002 & -0.058 & 0.94 & $0.90,0.99$ & 0.01 & -0.055 & 0.95 & $0.90,0.99$ & 0.02 \\
\hline Fermented milk products (serving/week) & -0.027 & 0.97 & $0.95,0.99$ & 0.01 & -0.027 & 0.97 & $0.95,0.995$ & 0.01 & -0.028 & 0.97 & $0.95,0.99$ & 0.01 \\
\hline Total low-fat/fat-free dairy products (serving/week) $\|$ & -0.021 & 0.98 & $0.96,0.999$ & 0.04 & -0.020 & 0.98 & $0.96,1.00$ & 0.06 & -0.022 & 0.98 & $0.96,0.999$ & 0.04 \\
\hline Multiplicative term of low-fat/fat-free dairy products and follow-up time & 0.003 & 1.00 & $1.00,1.01$ & 0.07 & 0.003 & 1.00 & $1.00,1.01$ & 0.04 & 0.003 & 1.00 & $1.00,1.01$ & 0.04 \\
\hline Skimmed and low-fat milk (serving/week) $\|$ & -0.025 & 0.98 & $0.95,0.997$ & 0.03 & -0.025 & 0.98 & $0.95,0.998$ & 0.04 & -0.027 & 0.97 & $0.95,0.997$ & 0.02 \\
\hline Multiplicative term of skimmed/low-fat milk and follow-up time & 0.004 & 1.00 & $1.00,1.01$ & 0.02 & 0.004 & 1.00 & $1.00,1.01$ & 0.01 & 0.004 & 1.00 & $1.00,1.01$ & 0.01 \\
\hline Cream and butter (serving/week)\| & -0.025 & 0.98 & $0.96,0.99$ & 0.005 & -0.031 & 0.97 & $0.95,0.99$ & $<0.001$ & -0.029 & 0.97 & $0.95,0.99$ & 0.002 \\
\hline Multiplicative term of cream/butter and follow-up time & 0.002 & 1.00 & $1.00,1.01$ & 0.03 & 0.002 & 1.00 & $1.00,1.01$ & 0.04 & 0.003 & 1.00 & $1.00,1.01$ & 0.03 \\
\hline
\end{tabular}

* Dairy/milk products consumption was calculated by using the cumulative average consumption before the onset of incident hypertension or before the end of the study, and entered into models as a continuous, time-varying variable. Total dairy products included skimmed/low-fat milk, whole milk, sherbet/ice milk, ice cream, yoghurt, cottage/ricotta cheese and other cheese (e.g. American, Cheddar, etc.). Low-fat dairy products included skimmed/low-fat milk, sherbet/ice milk and yoghurt. High-fat dairy products included whole milk, ice cream, cottage/ricotta cheese and other cheese (e.g. American, Cheddar, etc.). Fluid milk includes skimmed/low-fat milk and whole milk. According to the definition by the US Department of Agriculture MyPlate Dairy Group ${ }^{(28)}$ and the Dietary Guidelines for Americans ${ }^{(29)}$, milk products with little to no Ca (e.g. cream cheese, cream and butter) are not included in the Dairy Group. Therefore, for the present study, we did not include cream, sour cream, cream cheese and butter in any of the dairy groups. However, for the purpose of comparison, we created a separate group 'Cream and butter' by summing the intake of these four milk products. In addition, the 'Fermented milk products' group includes sour cream, yoghurt, cottage/ricotta cheese, cream cheese and other cheese (e.g. American, Cheddar, etc.). For all dairy/milk products, we used unit 'servings' to cream $=12 \mathrm{~g} ; 1$ serving sherbetice milk =96 g: 1 serving ice cream $=66 \mathrm{~g} ; 1$ serving yoghurt $=227 \mathrm{~g} ; 1$ serving cottage/ricotta cheese $=105 \mathrm{~g} ; 1$ serving cream cheese $=28 \mathrm{~g} ; 1$ serving other cheese $(\mathrm{e} . \mathrm{g}$. American, Cheddar etc.) $=28 \mathrm{~g} ; 1$ serving butter $=5 \mathrm{~g}$.

† Model 1: adjusted for sex, baseline age; time-varying total energy intake; and the multiplicative term of follow-up time with age.

‡ Model 2: model 1 + time-varying smoking status, physical activity, modified Dietary Guidelines Adherence Index (DGAI) score (i.e. DGAl score excluding sub-scores for assessing the consumption amount of milk and milk products and the likelihood of choosing low-fat milk and milk products) and caffeine coffee intake; and the multiplicative terms of follow-up time with physical activity.

$\S$ Model 3: model $2+\mathrm{BMl}$ at the beginning of each exam interval.

|| The hazard of incident hypertension are non-proportional by the consumption of total low-fat/fat-free dairy foods, skimmed/low-fat milk or cream/butter (i.e. Cox proportional assumption is violated). 
known that yoghurt, despite being fermented from milk, contains approximately $50 \%$ more $\mathrm{K}$, Ca and $\mathrm{Mg}$ and $30 \%$ more protein in per $227 \mathrm{~g}$ serving than milk ${ }^{(27)}$. The nutrients in yoghurt may also be more bioavailable than in other dairy products ${ }^{(45)}$; and probiotics abundant in yoghurt ${ }^{(46)}$ but not in unfermented dairy products have been found to inhibit angiotensin-converting enzyme, and thus reduce $\mathrm{BP}^{(12)}$. These unique aspects of yoghurt may partially explain the different relationships observed with incident HTN. In a post hoc sensitivity analysis, we excluded yoghurt from total low-fat dairy group and found that the reduced total low-fat dairy group was no longer associated with DBP change or HTN risk (data not shown). This suggests that yoghurt is an important contributor to the total low-fat dairy group.

Some previous studies have reported that obesity may be a significant player in the association between dairy intake and BP and HTN. For instance, obesity may raise the activity of adipose renin-angiotensin system, which further enhances the synthesis of angiotensin II, and results in vasoconstriction ${ }^{(47)}$. In a previous study with the same FHS cohort as the present study, we found that the consumption of yoghurt but not skimmed/low-fat milk was associated with a smaller annualised gain in body weight and waist circumference ${ }^{(48)}$, which may further explain the benefit of yoghurt over skimmed/low-fat milk. However, it should be noted that the adjustment of timevarying BMI or other metabolic factors did not substantially change the dairy foods-HTN associations found in the present study.

As most dairy fats are SFA that are generally considered to be atherogenic $^{(49)}$, low-fat and fat-free dairy foods are recommended in the 2010 Dietary Guidelines for Americans in order to provide adequate nutrients while limiting SFA and energy intake $^{(29)}$. In line with this recommendation, we found that higher intakes of low-fat/fat-free dairy foods were associated with smaller annualised increment in SBP and DBP. However, the health impact of high-fat dairy foods remains uncertain. Several studies have reported that the blood levels of dairyspecific fatty acids, as approximate biomarkers for dairy intake, were inversely related to inflammation, myocardial infarction, stroke and other disease conditions ${ }^{(16,40,50,51)}$. In a recent 10-year longitudinal study among 5209 middle-age to old adults, higher intake of SFA from dairy foods was related to lower CVD risk, whereas a higher CVD risk was found with increasing intake of meat-source SFA ${ }^{(14)}$. Dairy fats are the primary food source of CLA, which may lower BP by reducing the adipocyte-released vasoactive molecules ${ }^{(52)}$, whereas the potential synergism of various nutrients in dairy products may also contribute to its health benefits. Although high-fat dairy (e.g. whole milk, cheese) consumption was not associated with BP change in the present study, the significantly inverse association found between total high-fat dairy intake and HTN incidence in the discrete-time hazard model may indicate that keeping up an appropriate level of high-fat dairy consumption in the context of an energy-balanced, healthy dietary pattern may have a small, cumulative benefit on HTN prevention over the long run.

This study has several strengths such as the prospective design with approximately 15 years of follow-up and a relatively large sample size, repeated measurements of both outcome and exposure variables, exploration of various forms of dairy and milk products and consideration of both of BP and incident HTN in different statistical models with or without accounting for followup time. However, residual confounding factors cannot be completely ruled out. There also may be some misclassification due to the assumptions made on the type of dairy foods (e.g. yoghurt assumed to be low-fat). Finally, the results of this study may not be generalised to populations other than Caucasians, given that most of our participants were of European descent.

In summary, this longitudinal study with approximately 15 years of follow-up found an inverse association of dairy intake with average BP change per year and the long-term development of incident HTN. Although it merits further confirmation, these observations support the potential benefit of dairy products on BP management and incidence of HTN.

\section{Acknowledgements}

The authors thank Kara A. Livingston and Gail T. Rogers, Jean Mayer US Department of Agriculture Human Nutrition Research Center on Aging at Tufts University, for the help with data set management.

This work was supported by NHLBI contract no. NO1-HC-25195, US Department of Agriculture Agreement 58-1950-0-104 and research grants from The Dannon Company Inc. and General Mills Bell Institute of Health and Nutrition. Any opinions, findings, conclusions or recommendations expressed in this paper are those of the authors and do not necessarily represent those of the NHLBI, the National Institutes of Health, the US Department of Health and Human Services or the US Department of Agriculture. The Dannon Company Inc. and General Mills Bell Institute of Health and Nutrition had no role in the design, analysis or writing of this article.

Designed research: P. F. J and N. M. M.; conducted research: P. F. J. and H. W.; provided essential reagents or provided essential materials: C. S. F.; analysed data or performed statistical analysis: H. W.; wrote the paper: H. W. and P. F. J.; had primary responsibility for the final content: P. F. J.; other: critical review: L. M. T., N. M. M. and C. S. F.

P. F. J. and H. W. received support from a grant from The Dannon Company Inc. P. F. J. is a member of the Dannon Yogurt Advisory Board. N. M. M. and L. M. T. were funded in part by a grant from General Mills Bell Institute of Health and Nutrition. C. S. F. has no conflict of interests to declare.

\section{Supplementary material}

For supplementary material/s referred to in this article, please visit http://dx.doi.org/doi:10.1017/S0007114515003578

\section{References}

1. Chobanian AV, Bakris GL, Black HR, et al. (2003) The Seventh Report of the Joint National Committee on Prevention, Detection, Evaluation, and Treatment of High Blood Pressure: the JNC 7 report. JAMA 289, 2560-2572. 
2. Olives C, Myerson R, Mokdad AH, et al. (2013) Prevalence, awareness, treatment, and control of hypertension in United States counties, 2001-2009. PLOS ONE 8, e60308.

3. Kearney PM, Whelton M, Reynolds K, et al. (2005) Global burden of hypertension: analysis of worldwide data. Lancet 365, 217-223.

4. Vasan RS, Beiser A, Seshadri S, et al. (2002) Residual lifetime risk for developing hypertension in middle-aged women and men: the Framingham Heart Study. JAMA 287, 1003-1010.

5. Bertoia ML, Waring ME, Gupta PS, et al. (2011) Implications of new hypertension guidelines in the United States. Hypertension 58, 361-366.

6. Mancia G (2007) Blood pressure reduction and cardiovascular outcomes: past, present, and future. Am J Cardiol 100, 3J-9J.

7. Law M, Wald N \& Morris J (2003) Lowering blood pressure to prevent myocardial infarction and stroke: a new preventive strategy. Health Technol Assess 7, 1-94.

8. American Heart Association Nutrition Committee, Lichtenstein AH, Appel LJ, et al. (2006) Diet and lifestyle recommendations revision 2006: a scientific statement from the American Heart Association Nutrition Committee. Circulation 114, 82-96.

9. Appel LJ, Brands MW, Daniels SR, et al. (2006) Dietary approaches to prevent and treat hypertension: a scientific statement from the American Heart Association. Hypertension 47, 296-308.

10. McGrane MM, Essery E, Obbagy J, et al. (2011) Dairy consumption, blood pressure, and risk of hypertension: an evidence-based review of recent literature. Curr Cardiovasc Risk Rep 5, 287-298.

11. Park KM \& Cifelli CJ (2013) Dairy and blood pressure: a fresh look at the evidence. Nutr Rev 71, 149-157.

12. Ralston RA, Lee JH, Truby H, et al. (2011) A systematic review and meta-analysis of elevated blood pressure and consumption of dairy foods. J Hum Hypertens 26, 3-13.

13. Soedamah-Muthu SS, Verberne LD, Ding EL, et al. (2012) Dairy consumption and incidence of hypertension: a dose-response meta-analysis of prospective cohort studies. Hypertension 60, 1131-1137.

14. de Oliveira Otto MC, Mozaffarian D, Kromhout D, et al. (2012) Dietary intake of saturated fat by food source and incident cardiovascular disease: the Multi-Ethnic Study of Atherosclerosis. Am J Clin Nutr 96, 397-404.

15. German JB, Gibson RA, Krauss RM, et al. (2009) A reappraisal of the impact of dairy foods and milk fat on cardiovascular disease risk. Eur J Nutr 48, 191-203.

16. Wang H, Steffen LM, Vessby B, et al. (2011) Obesity modifies the relations between serum markers of dairy fats and inflammation and oxidative stress among adolescents. Obesity (Silver Spring) 19, 2404-2410.

17. Anderson AL, Harris TB, Tylavsky FA, et al. (2012) Dietary patterns, insulin sensitivity and inflammation in older adults. Eur J Clin Nutr 66, 18-24.

18. Shin S \& Joung H (2013) A dairy and fruit dietary pattern is associated with a reduced likelihood of osteoporosis in Korean postmenopausal women. Br J Nutr 110, 1926-1933.

19. Brunner EJ, Mosdol A, Witte DR, et al. (2008) Dietary patterns and $15-y$ risks of major coronary events, diabetes, and mortality. Am J Clin Nutr 87, 1414-1421.

20. Alonso A, Steffen LM \& Folsom AR (2009) Dairy intake and changes in blood pressure over 9 years: the ARIC study. Eur J Clin Nutr 63, 1272-1275.

21. Heraclides A, Mishra GD, Hardy RJ, et al. (2011) Dairy intake, blood pressure and incident hypertension in a general British population: the 1946 birth cohort. Eur J Nutr 51, 583-591.
22. Framingham Heart Study (2012) Framingham Heart Study. http://www.framinghamheartstudy.org/ (accessed February 2012).

23. Rimm EB, Giovannucci EL, Stampfer MJ, et al. (1992) Reproducibility and validity of an expanded self-administered semiquantitative food frequency questionnaire among male health professionals. Am J Epidemiol 135, 1114-1126; discussion $1127-1136$.

24. Willett WC, Reynolds RD, Cottrell-Hoehner S, et al. (1987) Validation of a semi-quantitative food frequency questionnaire: comparison with a 1-year diet record. J Am Diet Assoc 87, 43-47.

25. Feskanich D, Rimm EB, Giovannucci EL, et al. (1993) Reproducibility and validity of food intake measurements from a semiquantitative food frequency questionnaire. $\mathrm{J} \mathrm{Am}$ Diet Assoc 93, 790-796.

26. Salvini S, Hunter DJ, Sampson L, et al. (1989) Food-based validation of a dietary questionnaire: the effects of week-toweek variation in food consumption. Int $J$ Epidemiol 18, 858-867.

27. Agricultural Research Service, United States Department of Agriculture (2013) National Nutrient Database for Standard Reference, Release 26. http://ndb.nal.usda.gov/ndb/search/ list (accessed December 2013).

28. US Department of Agriculture (2015) What foods are included in the Dairy Group? http://www.choosemyplate.gov/dairy. html (accessed February 2015).

29. United States Department of Agriculture (2010) Dietary Guidelines for Americans (Policy Document). Released 31 January 2011. http://www.cnpp.usda.gov/Publications/ DietaryGuidelines/2010/PolicyDoc/PolicyDoc.pdf (accessed September 2012).

30. Fogli-Cawley JJ, Dwyer JT, Saltzman E, et al. (2006) The 2005 Dietary Guidelines for Americans Adherence Index: development and application. J Nutr 136, 2908-2915.

31. Chobanian AV, Bakris GL, Black HR, et al. (2003) Seventh report of the Joint National Committee on Prevention, Detection, Evaluation, and Treatment of High Blood Pressure. Hypertension 42, 1206-1252.

32. Kannel WB \& Sorlie P (1979) Some health benefits of physical activity. The Framingham Study. Arch Intern Med 139, 857-861.

33. World Health Organization (1980) WHO Expert Committee on Diabetes Mellitus: Second Report. Geneva: WHO.

34. McNamara JR \& Schaefer EJ (1987) Automated enzymatic standardized lipid analyses for plasma and lipoprotein fractions. Clin Chim Acta 166, 1-8.

35. Warnick GR, Benderson J \& Albers JJ (1982) Dextran sulfate$\mathrm{Mg}^{2+}$ precipitation procedure for quantitation of high-densitylipoprotein cholesterol. Clin Chem 28, 1379-1388.

36. Wang H, Livingston KA, Fox CS, et al. (2013) Yogurt consumption is associated with better diet quality and metabolic profile in American men and women. Nutr Res 33, $18-26$.

37. Engberink MF, Geleijnse JM, de Jong N, et al. (2009) Dairy intake, blood pressure, and incident hypertension in a general Dutch population. J Nutr 139, 582-587.

38. Dietary Guidelines Advisory Committee (2010) Report of the Dietary Guidelines Advisory Committee on the Dietary Guidelines for Americans, 2010, pp. 137-143. Washington, DC: US Department of Agriculture, Agricultural Research Service.

39. Pfeuffer M \& Schrezenmeir J (2007) Milk and the metabolic syndrome. Obes Rev 8, 109-118.

40. Mozaffarian D, de Oliveira Otto MC, Lemaitre RN, et al. (2013) trans-Palmitoleic acid, other dairy fat biomarkers, and incident 
diabetes: the Multi-Ethnic Study of Atherosclerosis (MESA). Am J Clin Nutr 97, 854-861.

41. Pimenta E \& Oparil S (2012) Management of hypertension in the elderly. Nat Rev Cardiol 9, 286-296.

42. Aronow WS, Fleg JL, Pepine CJ, et al. (2011) ACCF/AHA 2011 expert consensus document on hypertension in the elderly: a report of the American College of Cardiology Foundation Task Force on Clinical Expert Consensus Documents. Circulation 123, 2434-2506.

43. Livingstone KM, Lovegrove JA, Cockcroft JR, et al. (2013) Does dairy food intake predict arterial stiffness and blood pressure in men?: evidence from the Caerphilly Prospective Study. Hypertension 61, 42-47.

44. Crichton GE, Elias MF, Dore GA, et al. (2012) Relations between dairy food intake and arterial stiffness: pulse wave velocity and pulse pressure. Hypertension 59, 1044-1051.

45. Buttriss J (1997) Nutritional properties of fermented milk products. Int J Dairy Tech 50, 21-27.

46. Parvez S, Malik KA, Ah Kang S, et al. (2006) Probiotics and their fermented food products are beneficial for health. J Appl Microbiol 100, 1171-1185.
47. Engeli S, Negrel R \& Sharma AM (2000) Physiology and pathophysiology of the adipose tissue renin-angiotensin system. Hypertension 35, 1270-1277.

48. Wang H, Troy LM, Rogers GT, et al. (2013) Longitudinal association between dairy consumption and changes of body weight and waist circumference: the Framingham Heart Study. Int J Obes (Lond) 38, 299-305.

49. Astrup A, Dyerberg J, Elwood P, et al. (2011) The role of reducing intakes of saturated fat in the prevention of cardiovascular disease: where does the evidence stand in 2010? Am J Clin Nutr 93, 684-688.

50. Warensjo E, Smedman A, Stegmayr B, et al. (2009) troke and plasma markers of milk fat intake - a prospective nested case-control study. Nutr J 8, 21.

51. Warensjo E, Jansson JH, Cederholm T, et al. (2010) Biomarkers of milk fat and the risk of myocardial infarction in men and women: a prospective, matched case-control study. Am J Clin Nutr 92, 194-202.

52. DeClercq V, Taylor CG \& Zahradka P (2012) Isomer-specific effects of conjugated linoleic acid on blood pressure, adipocyte size and function. Br J Nutr 107, 1413-1421. 